\title{
Performance analysis on transmission of multilevel optical pulses using absolute polar duty cycle division multiplexing
}

\begin{abstract}
In order to explore the potential of optical multilevel signaling for high speed optical fiber networks, an absolute polar duty cycle division multiplexing (AP-DCDM) is demonstrated. Three users, each with the data rate of $10 \mathrm{~Gb} / \mathrm{s}$ were successfully multiplexed and transmitted over a single WDM channel, which can offer a possible transmission rate of $30 \mathrm{~Gb} / \mathrm{s}$ per WDM channel. The performance of AP-DCDM technique is examined, with comparison to $30 \mathrm{~Gb} / \mathrm{s}$ Time Division Multiplexing (TDM). Back-to-back receiver sensitivity of $-29.2 \mathrm{dBm}$ with OSNR of $22.5 \mathrm{~dB}$ was achieved for the worst user, together with the chromatic dispersion tolerance ranging from $207 \mathrm{ps} / \mathrm{nm}$ to $276 \mathrm{ps} / \mathrm{nm}$. A comparison with conventional TDM technique shows a clear advantage of the proposed AP-DCDM technique.
\end{abstract}

